\title{
A CLUSTER BASED INTELLIGENT METHOD TO MANAGE LOAD OF CONTROLLERS IN SDN-IOT NETWORKS FOR SMART CITIES
}

\author{
SURENDRA KUMAR KESHARI* VINEET KANSAL ${ }^{\dagger}$ AND SUMIT KUMAR ${ }^{\ddagger}$
}

\begin{abstract}
Software Defined Network (SDN) is a programmable network which separates the control logic-plane and hardware data-plane. The SDN centrally manages different Internet of Things (IoT) enabled smart devices like, actuators and sensors connected in the networks. Smart city infrastructure is an application of IoT network which purpose is to manage the city network without human interventions. To collect the real time data, such smart devices generate large amount of data and increasing the traffic in network. To maintain the quality of services (QoS) of smart city IoT networks, the SDN needs to deploy the multi-controllers. But the communication performance reduces due to unbalance load distribution on controllers. To balance the traffic load of controller an intelligent cluster based Grey Wolf Optimization Affinity Propagation (GWOAP) Algorithm is proposed when deploying the multiple controllers in SDN-IoT enabled smart city networks. The proposed algorithm is simulated and the experimental results able to calculates the minimum overall communication cost in comparison with Genetic Algorithm (GA), Particle Swarm Optimization (PSO) and Affinity Propagation (AP). The proposed GWOAP better balance the IoT enabled smart switches among clusters and node equalization is balanced for each controller in deployed topology. By using the proposed methodology, the traffic load of IoT enabled devices in smart city networks intelligently better balance among controllers.
\end{abstract}

Key words: Controller, CPP, IoT, SDN, optimization, smart city.

AMS subject classifications. 68M14

1. Introduction. The new paradigms of programmable networking infrastructure is becoming popular because of its separation feature of data plane and control plane [1]. Such networks facilitate the flexible management in a complex large, distributed network. Software defined infrastructure centrally monitors the devices like, sensor and actuator which are connected in IoT networks to process real time data. The smart networking is significantly promoted by Software defined infrastructure due to limitation of existing IoT networking like, security issues, device management, configurations management and flexibility [2]. As number of smart devices increasing in smart city IoT network, the flow of heavy traffic are also increasing.

The smart cities are employed with the varieties of SDN-IoT networks by using with artificial intelligence in mobile networking, automatic traffic monitoring, streaming of videos, smart surveillances, secure communication and e-healthcare [3] [4] [5]. Such applications required high volume of real time data transmission with low latency. The traditional network environment is unable to meet the processing of such real time data with minimum delay. So, the SDN infrastructure is the promising solution of real time data processing in smart city networks.

For the number of real time devices in smart cities infrastructure a single controller is not sufficient. So, more controllers are needed to place to manage such SDN-IoT network [6]. But multiple controller placements are NP-hard problem with the issue of required number of controller and controller placement at optimal place to balance the traffic load at minimum latency. Still there are few challenges like, optimization of the multiple controllers and minimize the propagation delays in such networking so that costs may be reduced and performance may be improved [7]. The Controller Placements Problem(CPP) is described by Heller et.al. [8] and calculated the average delays between controller and switches. Jalili et al. [9] described the controllers

\footnotetext{
*Department of Information Technology, KIET Group of Institutions, Delhi-NCR, Ghaziabad, Dr. A.P.J. Abdul Kalam Technical University, Lucknow, India (surendra.keshari@kiet.edu).

${ }^{\dagger}$ Department of Computer Science and Engineering, Institute of Engineering and Technology, Dr. A.P.J. Abdul Kalam Technical University, Lucknow, India (vineetkansal@yahoo.com).

${ }^{\ddagger}$ Department of Computer Science and Engineering, Amity School of Engineering and Technology, Amity University, Noida, India (sumitkumarbsr19@gmail.com).
} 
placements problems and proposed a multi objective Genetic Algorithm (GA) that yield low memory space and better computations time. There are many optimization methods applied to solve such complex issues like, CPP. Gao et. al. [10] and K.S.Sahoo et. al. [11] studied the some optimization methods to maximizes the network survivability and minimize the latency and also suggested the Particle Swarm Optimization (PSO) based mechanism to solve the problem of CPP and managed the dynamic load over capacitated controllers.

Few researchers studied the multiple controller deployments approaches. To improve the controller's reliability and survivability in SDN, the multi controller's deployments strategies suggested based on capacitated controllers' and multi-path diversity method [15] [16] [17].

The current research works of CPP in programmable network concludes the multi controller deployments models which are commonly based on communication delays between switch to controller and controller to controller latencies. Such calculations of the communication delays are based on different optimization models.

Grey Wolf Optimization (GWO) [18] hunt the preys by using the supportive technique. This search method based on the hunting behaviour of grey wolves in nature. The wolf alpha $(\alpha)$ is the leader and beta $(\beta)$ and delta $(\delta)$ follow the wolf alpha $(\alpha)$. The remaining wolves in pack are represented as omega $(\omega)$. Each wolf guides their subsequent wolf in the hierarchy to hunt the prey. The clustering algorithm Affinity Propagation (AP) [19] is based on similarities and data points between pairs. The similarities serve as the cluster centre and is selected by the greater value of similarities. The advantage of this algorithm is need not to initialized the cluster centre and cluster numbers.

The main contributions of the paper are:

- An intelligent cluster-based load balancing Grey Wolf Optimization Affinity Propagation Algorithm (GWOAP) is proposed to deploy the controller in SDN-IoT enabled smart city networks.

- An effective fitness function is proposed to minimize the overall communication cost in SDN-IoT networks.

The rest of paper is summarized as follows. In section 2, the related works are summarized, In section 3 , the problem is mathematically formulated. Section 4, Discussed the related algorithm, Section 5, illustrates the network model and proposed GWOAP algorithm. The section 6 illustrates the result and discussion. Finally, section 7 concludes the paper.

2. Related Works. The benefits of Software Defined Networks always attracted to researchers in recent few years. The programmable network SDN centrally manages different IoT devices connected in the networks. SDN-IoTs network has significant attentions in both academia and industry.

Heller et. al. [8] introduced the Controller Placements Problem to decide how many and where controllers to be placed in SDN to achieve scalability. Author also discussed the challenges of CPP for each switch node and threshold of communication latency and fault tolerance.

Gao et. al. [10] suggested the PSO based methodology to solve the Controller Placement Problem and manage the dynamic load of controllers. Sahoo et. al. [11] proposed the CPP_PSO and CPP_FFA optimization methodology that minimizing the latency and maximizing the network survivability. Author also proposed a fitness functions to focused the network survivability which designed for reliable control plane.

Jimenez et. al. [12] used the K-critical methodology to deploy the minimum controller in the networks and found robust controller deployments. Ishigaki et. al. [13] suggested a controller deployment methodology based on node calculations index. Zhao et al. used affinity propagation to modify their methodology and suggested new clustering algorithm. Zhao [14] evaluated their algorithm and found better networks performance when compared with other clustering algorithms. Sallahi et. al. [20] evaluated the controller deployment cost and suggested a multi controller deployment model. Wang et. al. [21] used the capacitated controllers' approach to balance the load of controllers and followed the multi controller deployment strategies .

A small area network is efficiently manageable by a single controller due to its less overhead in network congestion. But for large area network the overhead of single controller increased and is insufficient to manage their network [22].

For a distributed Software Defined-IoT network to manage the flow of data multiple controllers are needed but the issue of multiple controller placements is challenging [23].

Onix [24], Hyperflow [25], and Devoflow [26] were used as the distributed control architecture to solve the problem of reliability and scalability of SDN. 
ONOS [27] and Open Daylight [27] are open-source controllers and used in large data centres and in WAN. These controllers are capable to control and manage the large data flow of IoT devices in smart city networks. A POCO model is suggested to consider multiple controller deployments optimizations. The Pareto methodology included the controller overhead and latency delay to consider the multiple controller placements optimization problems [28].

Liu et. al. [29] considered the propagation latencies, controller's load and suggested the networks clusters using PSO methodology . Beheshti et. al. [30] studied the SDN reliability as well as resiliency and evaluate the controller-switch performance. To deploy the controller heuristic approach is suggested to consider the reliability as well as resiliency in SDN network . Killi et al. [31] proposed the game theory and k-means partitioning method to place the multiple controller in large distributed network.

Keshari et al. [32] proposed an intelligent method to place the controller in SDN-IOT network. Here, author addressed the dynamic controller placement issue but when nodes increase the load balancing issue arises. Smart city network is employed with large IoT network infrastructure and to improve the better networking experience the virtual network placements are needed to optimize [33] [34] [35].

The above literature focuses on controller deployment only. Few researchers used partitioning clustering but dynamically load management is not suitable. Therefore, a cluster-based approach by using affinity propagation method is proposed where the cluster centre need not to initialize. In this paper, GWOAP algorithm is proposed to deploy the controller and dynamically mange the load of controllers.

3. Problem formulation. The network topology can be depicted like undirected graph $G(N, L)$, here $N$ represent the set of forwarding node and $L$ represent a link set. The node set consists of the switches and controllers.

Let $d(n, c)$ denotes the shortest distance paths between node $n \in N$ and controller $c \in C$. C denotes a set of all possible controllers, $C=\left\{c_{1}, c_{2}, \ldots . . c_{x}\right\}$.

Let $d\left(c_{i}, c_{j}\right)$ be the shortest path between controller $c_{i}$ to controller $c_{j}$ where $c_{i}, c_{j} \in C$.

If any $i^{\text {th }}$ switch node is connected to a $j^{\text {th }}$ controller node then the distance between $i^{\text {th }}$ switch node and $j^{\text {th }}$ controller node is $d_{i j}(i, j)$. The binary value $z_{i j}=1$, which means $i^{t h}$ switch is successfully connected to $j^{\text {th }}$ controller.

Then shortest distance between $i^{t h}$ switch node and $j^{\text {th }}$ controller node is:

$$
z_{i j}=\left\{\begin{array}{l}
1: \text { when switch } i \text { is connected to controller } j \\
0: \text { otherwise }
\end{array}\right.
$$

Every controller in the network topology processes the request flow for all connected switches. The total traffic load processed by $j^{\text {th }}$ Controller at time $\mathrm{t}$ is equal to sum of traffic flow by all connected switches in domain. Then,

$$
\Theta_{j}^{t}=\sum_{i=1}^{N} l_{i j}^{t}
$$

where, $l$ is the load of $i^{\text {th }}$ switch at time $t$. The processing capacity i.e. flow rate $(\mathrm{kb} / \mathrm{s})$ of controller is consider as $A_{j}$. Then, by the Queuing theory [36] and Littile's Law [37].

The mean waiting time to process the flow by the $j^{\text {th }}$ controller is:

$$
W_{j}^{t}=\frac{1}{A_{j-} \Theta_{j}^{t}}
$$

If the total node $|V|=|N|+|C|$ then the total average response time for $j^{\text {th }}$ controller is:

$$
\Delta t_{j}=|V|^{2} * W_{j}^{t}
$$

$\Delta t_{j}$ is considered as average processing times for $j^{t h}$ controller. So, at time $t$, the average controller's response times for the controller and switch is:

$$
T^{t}=\frac{\sum_{j=1}^{c} \theta_{j}^{t} * \Delta t_{j}}{\sum_{j=1}^{c} \theta_{j}^{t}}
$$


To calculate the total load cost, we have to find out the individual cluster domain cost i.e. intra domain cost and inter domain cluster cost. Considering here intradomain constant flow rate is $v$.

Then intra domain cost is:

$$
T_{\text {intrad }}=2 * v \sum_{j \in c} \sum_{i \in N}\left(\left|\frac{l_{i}^{t}}{v}\right| d_{i j} * z_{i j}^{t}\right)
$$

And inter domain cost is:

$$
T_{\text {interd }}=v \sum_{j \in c} \sum_{k \in c} d_{j k}
$$

So, Overall Cost:

$$
\text { Total }_{\text {min }}=\gamma * T_{\text {intrad }}+(1-\gamma) * T_{\text {interd }}
$$

Where, the weight factor , controls the inter domain and intra domain communications cost.

4. Related algorithms. Grey Wolf Optimization (GWO) [18] follow supportive techniques to hunt the preys. The search method in GWO algorithm is based on the grey wolves hunting behaviours as observed in the nature. There is wolf alpha $(\alpha)$ who is the leader of their pack, then there are another supportive leaders beta $(\beta)$ and then delta $(\delta)$ who follow the wolf alpha $(\alpha)$. The rest wolves are considered as omega $(\omega)$. Every wolf has their own responsibility which is represented as hierarchical structure that leader alpha $(\alpha)$ is on top position that provides the first solution. Other wolves respectively, beta $(\beta)$, delta $(\delta)$ and omega $(\omega)$ provides the subsequently second, third and final solution. Lastly, omega $(\omega)$ wolves are guided by their previous three leaders.

When all wolves find the prey, then there are three variables of coefficients that are enclosed with prey and use the encircling mechanisms.

The encircling mechanisms defined by following equations:

$$
\begin{aligned}
& D_{\alpha}^{\prime}=\left|C_{1}^{\prime} \cdot X_{a}^{\prime}-X_{i}^{\prime}\right| \\
& D_{\beta}^{\prime}=\left|C_{2}^{\prime} \cdot X_{\beta}^{\prime}-X_{i}^{\prime}\right| \\
& D_{\delta}^{\prime}=\left|C_{3}^{\prime} \cdot X_{\delta}^{\prime}-X_{i}^{\prime}\right|
\end{aligned}
$$

$X^{\prime}$ is the symbolizes the position vectors for the grey wolves and denotes the iterations. Then, $X_{i}^{\prime}$ denotes different wolves $\alpha, \beta$ and $\delta$.

$$
\begin{aligned}
& X_{1}^{\prime}=X_{\alpha}^{\prime}-A_{1} \cdot D_{\alpha}^{\prime} \\
& X_{2}^{\prime}=X_{\beta}^{\prime}-A_{2} \cdot D_{\beta}^{\prime} \\
& X_{3}^{\prime}=X_{\delta}^{\prime}-A_{3} \cdot D_{\delta}^{\prime}
\end{aligned}
$$

and

$$
\begin{gathered}
X_{i+1}^{\prime}=\left(X_{1}^{\prime}+X_{2}^{\prime}+X_{3}^{\prime}\right) / 3 \\
C=2 r_{2} \\
A=2 * a \cdot r_{1}+a
\end{gathered}
$$

$C$ and $A$ are coefficients vector, whereas $a$ is the controlling variable parameter to change the coefficient $A$. 
1. Initializes the populations $X_{m}^{\prime},(m=1,2, \ldots \ldots, n), X_{\alpha}^{\prime}, X_{\beta}^{\prime}$, and $X_{\delta}^{\prime}$

2. Set the $\mathrm{C}$ and $\mathrm{A}$ vectors.

3. While $(i<M a x i)$

4. For every search agents

5. update present locations search agents.

6. End For

7. Update A, C and a

8. Evaluating the fitness function

9. Update $X_{\alpha}^{\prime}, X_{\beta}^{\prime}$, and $X_{\delta}^{\prime}$

10. $i=i+1$

11. End while

12. $\operatorname{return} X_{\alpha}^{\prime}$

Affinity Propagation (AP) [19] is the clustering algorithm based on data point as input and similarities between pairs. By using the negative Euclidean distance, first denotes the similarities between available data point.

Suppose, $x_{i}=x_{1}, x_{2}, \ldots \ldots, x_{m}$ is data set for m objects. The Similarity is

$$
S(i, j)=-\left\|x_{i}^{2}-x_{j}^{2}\right\|
$$

where $i, j \in[1, m]$.

The similarities represent the degrees for a node to serve as the cluster center. The cluster center is selected by the greater value of similarities. In AP algorithm, there is no need to initialized the cluster center and cluster numbers. Here the preference needs to specify in advance and that is set to constant.

The availability and responsibility are calculated by AP algorithm for each node. If $i$ node denotes the cluster center and $j$ node denotes the responsibility. Then, the availability degree is represented by the node $i$ selecting cluster center to node $j$ :

$$
\begin{gathered}
r(i, j)= \begin{cases}S^{\prime}(i, j)-\max _{j \neq k} a(i, k)+S^{\prime}(i, k) & (i \neq j) \\
S^{\prime}(i, j)-\max _{j \neq k} S^{\prime}(i, k) & (i=j)\end{cases} \\
a(i, j)= \begin{cases}\min \left\{0, r(i, j)+\sum_{k \neq i, j} \max [0, r(k, j)]\right\} & (i \neq j) \\
\sum_{k \neq j} \max [0, r(k, j)] & (i=j)\end{cases}
\end{gathered}
$$

The search follows the iterative processes in AP algorithm to assemble the exemplar clusters.

To avoid oscillation, $\lambda$ introduced as a damping factor for availability and responsibility values. For the fast iteration and strong global searches, the smaller value is capable in AP. After continuous iterations, the AP select the cluster centre to the largest node. The iteration processes,

$$
\begin{aligned}
& r_{t}(i, j)=(1-\lambda) r_{t}(i, j)+\lambda r_{t-1}(i, j) \\
& a_{t}(i, j)=(1-\lambda) a_{t}(i, j)+\lambda a_{t-1}(i, j)
\end{aligned}
$$

and cluster centre

$$
c_{i}=\arg \max \{r(i, j)+a(i, j)\}
$$

5. Network model and proposed methodology. The proposed network model is illustrated in fig. 5.1. Here the controllers are deployed in a distributed environment and forms the clusters to balance the load of controllers. The figure shows that there are four controllers $C=\{C 1, C 2, C 3, C 4\}$ and fifteen switch nodes $N=\{S 1, S 2, ., S 15\}$ are deployed. Different number of switch nodes are assigned to different controllers. This situation leads to generate the unbalanced scenario among controllers. Thus, the controller deployment cost 


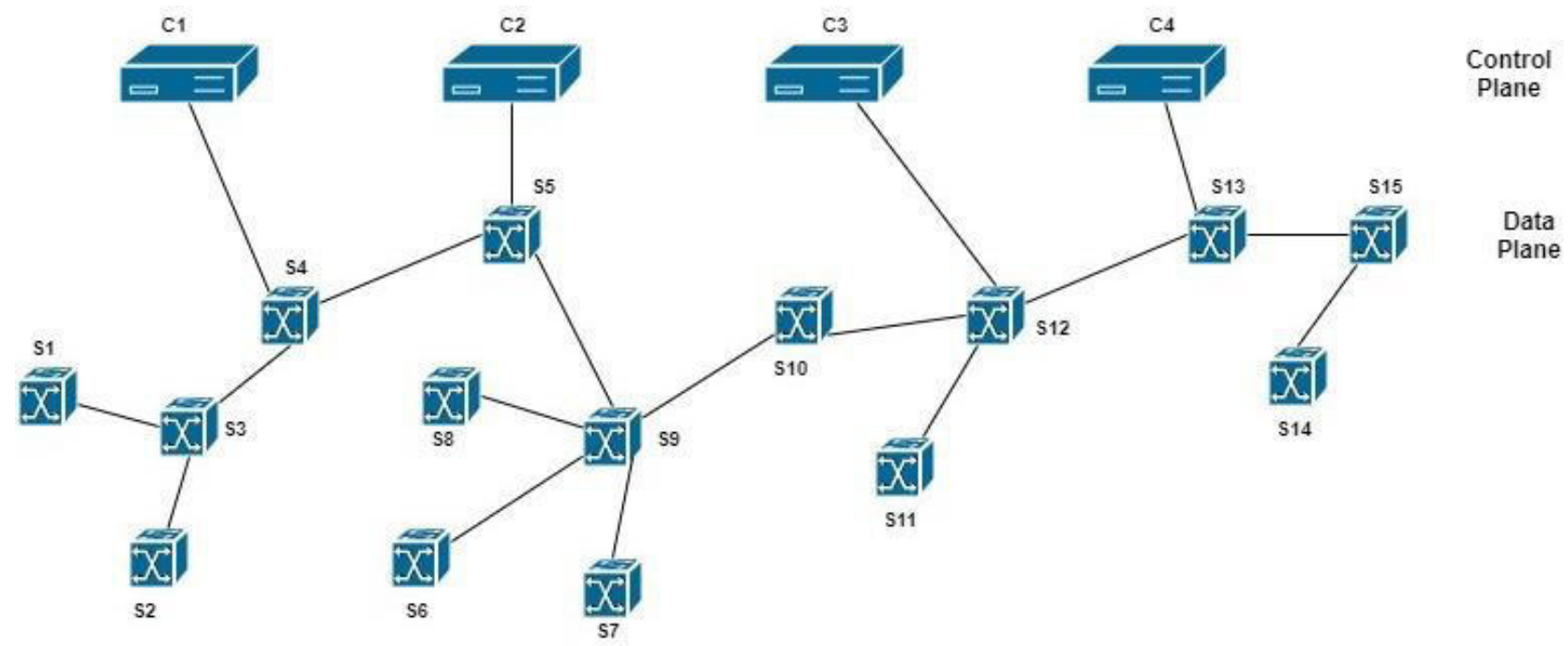

FIG. 5.1. Multi-controller deployment and cluster assignment

will be increased and affect overall communication cost to flow the traffic. Therefore, the proposed methodology solved such SDN problems and it maps the switch nodes to respective controller in the network. In the proposed methodology, the GWOAP algorithm forms the clusters of nodes by calculating the distance among switches and then assigned each cluster to respective controller by calculating the distance between cluster head to respective nearest controller. As given in fig. 5.1, the four switch nodes $\mathrm{S} 1, \mathrm{~S} 2, \mathrm{~S} 3, \mathrm{~S} 4$ forms the cluster-1 and assigned the load of cluster to controller C1. Similarly, the five switch nodes S5,S6,S7,S8,S9 forms the cluster-2 and assigned the load of cluster to controller C2. The three switch nodes S10,S11,S12 forms the cluster-3 and assigned the load of cluster to controller C3. Finally, the three switch nodes S13,S14,S15 forms the cluster-4 and assigned the load of cluster to controller $\mathrm{C} 4$. The switch nodes S4,S5,S12 and S13 are assigned the cluster head in their respective cluster. The methodology, forms four clusters and assigned to respective four deployed controllers.

Proposed Grey Wolf Optimization Affinity Propagation (GWOAP) Algorithm.

1. Extract and input the nodes from the Topology G (N, L).

2. Traverse the all nodes and calculate the request rate of switch.

3. Initialize the similarity matrix.

4. Update the AP parameters until condition reached.

5. Calculate the Cluster matrix.

6. Initialize grey wolf population $X_{m}(m=1,2, \ldots . n), X_{\alpha}^{\prime}, X_{\beta}^{\prime}$ and $X_{\delta}^{\prime}$

7. Set the $\mathrm{C}$ and $\mathrm{A}$ vectors.

8. For every search agents calculate the fitness by equation 3.8 :

$X_{\alpha}^{\prime}$ - is the best search engine

$X_{\beta^{-}}^{\prime}$ is the second best search engine

$X_{\delta^{-}}^{\prime}$ is the third best search engine

9. While $(i<$ maxiteration $)$

10. Update present positions for search agents

11. Update A, C and a

12. For every search, evaluating the fitness function

13. Update $X_{\alpha}^{\prime}, X_{\beta}^{\prime}$, and $X_{\delta}^{\prime}$

14. End For

15. $\mathrm{i}=\mathrm{i}+1$

16. End while 
17. $\operatorname{return} X_{\alpha}^{\prime}$

The proposed methodology for GWOAP algorithm is illustrated in figure 5.2. The traffic load of data needed to manage in SDN-IoT enabled networks due to real time data collections. Here, the goal is to manage the total traffic load processed by IoT enabled nodes. Therefore, the proposed methodology solved such SDN problems and it maps the switch nodes to respective controller in the network. The methodology forms the clusters and assigns the balance traffic load to respective controllers. The proposed GWOAP algorithm is the hybridization of GWO and AP. Grey Wolf Optimization solves the controller placement problems whereas, affinity propagation forms the clusters according to traffic load. The benefit of using AP algorithm is that, there is need not initializing the number of clusters in advance. It calculates dynamically, and identify the cluster centre. The OS3E topology is taken to evaluate the methodology. The approach follows the total 34 nodes, where 29 nodes are taken as switch nodes and 5 controllers are taken as controller nodes to make clusters. The experimental results, calculated minimum overall cost communication cost by our defined objective function. The proposed GWOAP better balance the switches among clusters and node equalization is balanced for each controller as compare to GA, PSO and AP approaches.

6. Result and Discussion. The proposed methodology is simulated and evaluated on MATLAB $2019 \mathrm{~b}$. The OS3E topology is taken to evaluate the experiments. The approach follows the total 34 nodes, where 29 nodes are taken as switch nodes and 5 controllers are taken as controller nodes to make clusters. The switch nodes and controller nodes create 42 links in the network and each node is independent from other node in the proposed network model. The simulations are evaluated on the 64-bit machine of Intel Core i5-4-processor having Windows 10 operating system with 8 GB RAM.

For the objective function the weighting factor is set equal to 0.8 and flow rate $\mathrm{v}$ is set to $1 \mathrm{~Kb} / \mathrm{sec}$. The objective is to minimize the overall communication cost using GWOAP approach. The AP algorithm better balance the number of nodes among different clusters and switch nodes managed by controller nodes. The GWO algorithm better place the controllers to manage switches. In AP algorithm, without initializing the no. of controllers the CPP problem is solved. But the GA and PSO approaches, needs to initialized the no. of controllers using other clustering approaches.

In the OS3E network topology, we applied the GA, PSO, GAAP, PSOAP and GWOAP algorithms and found that GWOAP better balance the switches among clusters. Thus, we calculated minimum overall cost communication cost by our defined objective function.

In fig. 6.1, the objective function shows the overall communication cost by applying the GA, PSO, GAAP, PSOAP and GWOAP algorithms by simulating the OS3E network topology. This figure also clearly shows that by applying the GA and PSO algorithm up to 6 controller the communication cost decreases as number of controllers increases. But after 6 controller the cost varies up till controller 10 due to unbalanced load of switches. When using the optimization algorithms GA, PSO and GWO with AP, algorithm the clustering approach better balance the switches on controllers. As shown in fig. 6.1, that on OS3E topology on 5 controllers the proposed GWOAP method performs better and obtain the lowest total cost which is the optimal value.

Fig. 6.2 shows the number of switch nodes controlled by individual controller for all five algorithms, when deployment of OS3E topology network. The same number of controllers deployment are considered controlled by each controller. There are 29 nodes are taken as switch nodes and 5 controllers are taken as controller nodes to make clusters. The largest differences found using GA algorithm in which controller C1 load is almost double of $\mathrm{C} 2$ and $\mathrm{C} 4$ controller due to crossover mutation. The PSO algorithm improves little better as compare to GA where C1 and C5 controllers balance more switches as compared to C2 and C4. Here also C1 load is almost double of $\mathrm{C} 2$ and $\mathrm{C} 4$ controller.

The AP algorithm is hybridized with GA, PSO and GWO optimization algorithms. AP forms the clusters by calculating the distance between switch and controller. So, the initialization of number of controllers are not required here. The node equalization is increasing better by using GAAP, PSOAP and GWOAP algorithms. Fig. 6.2, clearly shows that the GWOAP better balance the switches among clusters and node equalization is similar for each controller in deployed topology.

Fig. $\quad 6.3$ is the iterations convergence diagram which compares the performance of taken all five algorithms. The execution times using GWOAP is better and optimal among all other taken algorithms. The objective 


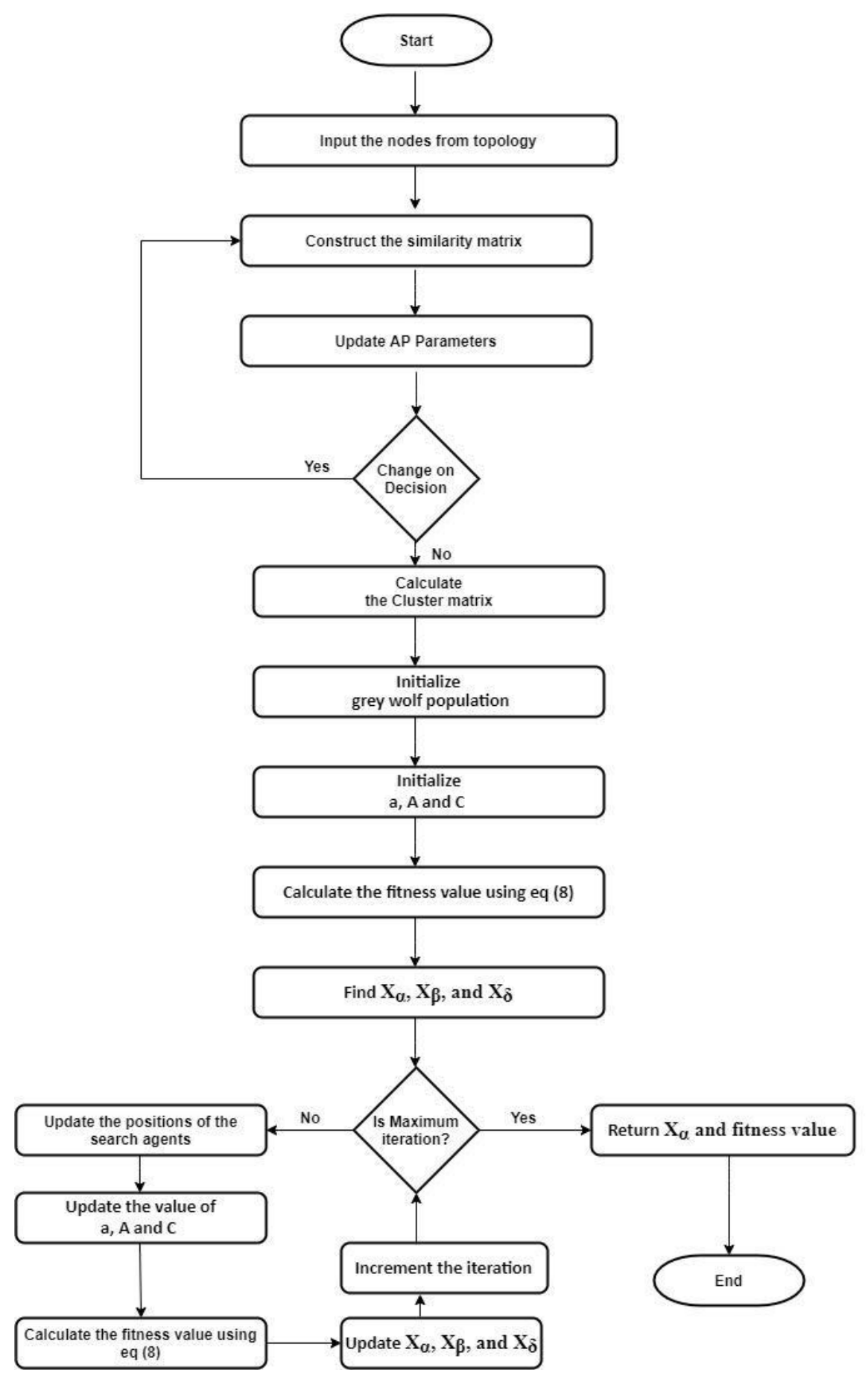

FIG. 5.2. Flow diagram of proposed GWOAP algorithm

function is iterated up to 300 executions to find optimal communication cost for taken algorithms.

The controller's deployments in the OS3E network topology divides the network into sub domains by the execution of AP algorithms and optimization algorithms optimize the processing delays as well as manage the controller's traffic load. 


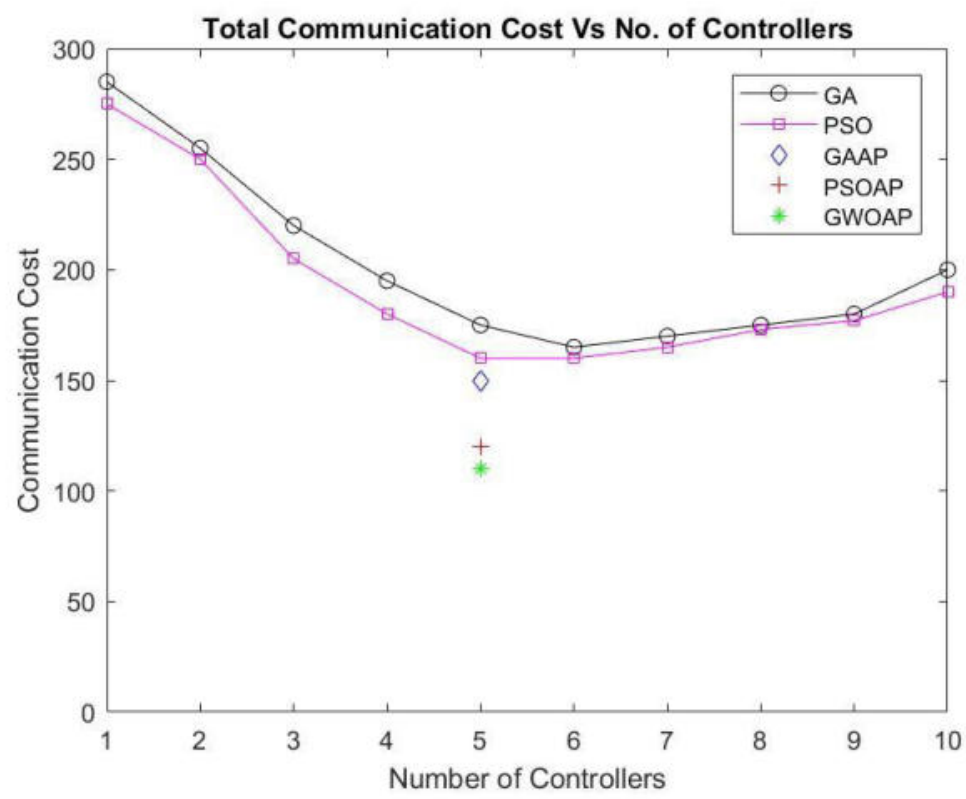

FIG. 6.1. Total Communication Cost Vs No. of Controllers

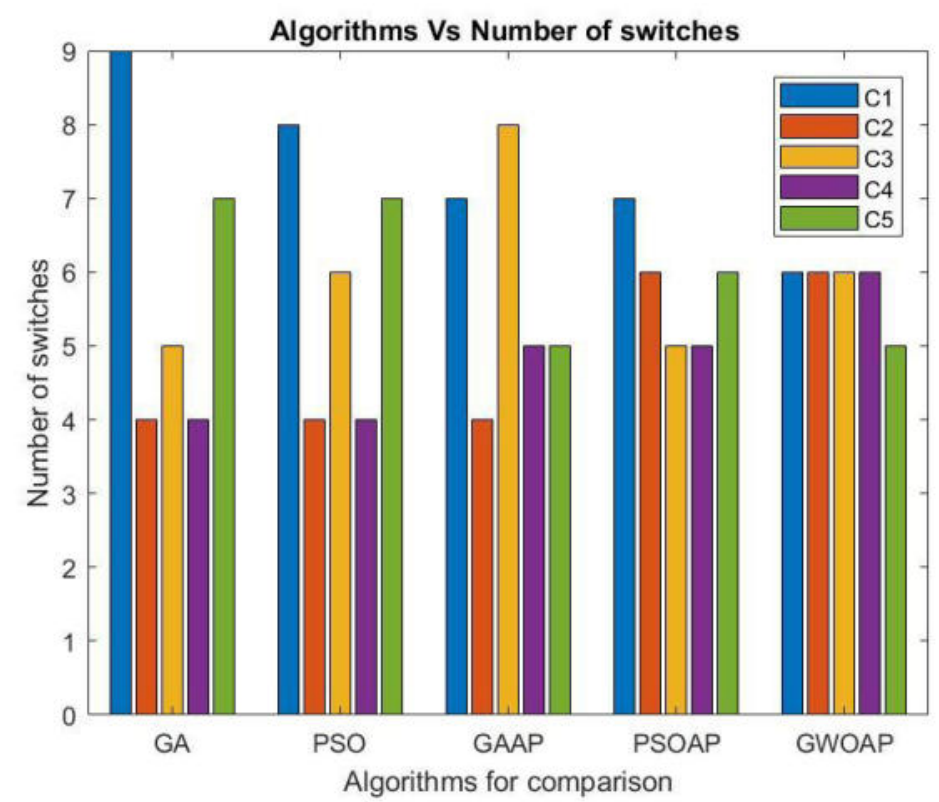

FIG. 6.2. Algorithm Vs Number of Switches

7. Conclusion. Software Defined Networking is a programmable network paradigm and having the advantages of it's separation of data plane and control plane. The programmable network SDN centrally manages different smart IoT devices connected in the networks. One of the emerging applications of IoT network is Smart City which purpose is to manage the city without human interventions.

This paper proposed a GWOAP algorithm for the placement of multi controllers in smart city networks. The controllers manage the traffic of connected smart devices in the network. The proposed algorithm is 


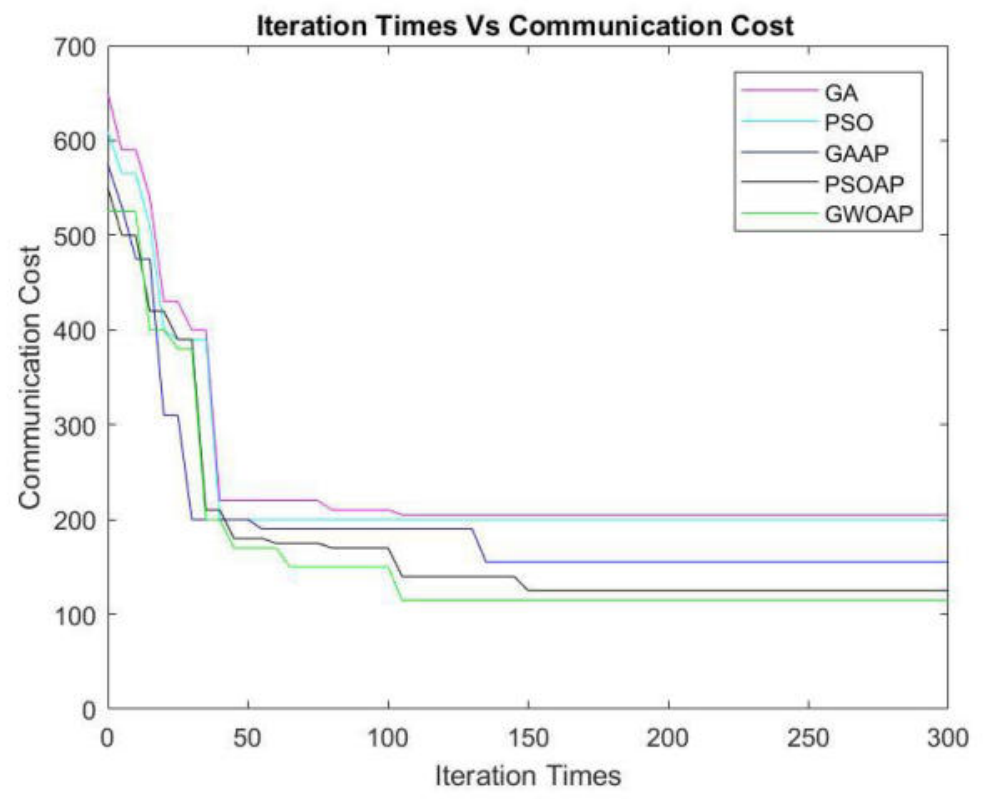

FIG. 6.3. Iteration Times Vs Communication Cost

simulated using OS3E network topology. The controller's deployments in the OS3E network topology divides the network into sub domains by the execution of AP algorithms and GWO optimization algorithms optimize the processing delays as well as manage the controller's traffic load.

The experimental results, calculated minimum overall cost communication cost by our defined objective function. GWOAP better balance the IoT enabled smart switches among clusters and node equalization is balanced for each controller in deployed topology. By using the proposed methodology, the traffic load of IoT enabled devices in smart city networks intelligently better balance among controllers.

\section{REFERENCES}

[1] Y. Zhang, L. CUI, W. WANG, AND Y. Zhang, A survey on software defined networking with multiple controllers, Journal of Network and Computer Applications, 103(2018) pp. 101-118.

[2] K. Sood, S. YU, AND Y. XIANG, Software-defined wireless networking opportunities and challenges for Internet-of-Things: A review, IEEE Internet Things J., vol. 3, no. 4, pp. 453-463, Aug. 2016.

[3] S. Sendra, A. Rego, J. Lloret, J.M. Jimenez, And O. Romero, Including artificial intelligence in a routing protocol using Software Defined Networks, IEEE International Conference on Communications Workshops, Paris, France, 21- 25 May 2017, pp. 670-674.

[4] M. TAha, L. GARCíA, J.M. Jimenez, AND J. LloRet, SDN-based throughput allocation in wireless networks for heterogeneous adaptive video streaming applications, 13th International Wireless Communications and Mobile Computing Conference, Valencia, Spain, 26-30 June 2017, pp. 963-968.

[5] X. XU, Q. HuAnG, AND X. Yin Intelligent offloading for collaborative smart city services in edge computing, IEEE Internet of Things Journal (2020).

[6] Wei Ren, Yan Sun, Hong Luo and Mohsen Guizani A Novel Control Plane Optimization Strategy for Important Nodes in SDN-IoT Networks, IEEE Internet of Things Journal, Vol. 6, No. 2, April 2019.

[7] S.K. Keshari, V. Kansal, And S. Kumar A Systematic Review of Quality of Services (QoS) in Software Defined Networking $(S D N)$, Wireless Personal Communications, Vol. 116, issue-3, pp. 2593-2614 (2021).

[8] B. Heller, R. Sherwood, And N. McKeown The controller placement problem. In: ACM; pp. 7-12, 2012.

[9] A. Jalili, V. Ahmadi, M. Keshtgari, and M. Kazemi Controller placement in software-defined WAN using multi objective genetic algorithm In: IEEE. pp. 656-662, 2015.

[10] C. Gao, H. Wang, F. Zhu, L. Zhai, And S. Yi A Particle Swarm Optimization Algorithm for Controller Placement Problem in Software Defined Network In: Springer, pp. 44-54, 2015.

[11] K.S.Sahoo, D.Puthal, And M.S. Obaidat On the placement of controllers in software-Defined-WAN using metaheuristic approach The Journal of Systems \& Software 145 (2018) pp. 180-194. 
[12] Y. Jimenez, C. Cervelló-Pastor, and A. J. Garcia On the controller placement for designing a distributed SDN control layer in Proc. Netw. Conf., Jun. 2014, pp. 1-9.

[13] G. Ishigaki and N. Shinomiya Controller placement algorithm to alleviate burdens on communication nodes in Proc. Int. Conf. Computer., Network. Communication, Feb. 2016, pp. 1-5.

[14] J. ZhaO, H. Qu, J. Zhao, Z. LuAn, AND Y. Guo Towards controller placement problem for software-defined network using affinity propagation Electron. Lett., vol. 53, no. 14, pp. 928-929, 2017.

[15] L. F. Müller, R. R. Oliveira, M. C. Luizelli, L. P. Gaspary, and M. P. Barcellos Survivor: An enhanced controller placement strategy for improving SDN survivability in Proc. IEEE Global Communication. Conf., Dec. 2014, pp. 19091915.

[16] Y. Hu,W.WAng, X. Gong, X. Que, AND S. Cheng On reliability-optimized controller placement for software-defined networks China Communication., vol. 11, no. 2, pp. 38-54, Feb. 2014.

[17] Guo, M. and Bhattacharya, P. Controller placement for improving resilience of software-defined networks In Proceedings of the 2013 Fourth International Conference on Networking and Distributed Computing (ICNDC), Los Angeles, CA, USA, 21-24 December 2013; pp. 23-27.

[18] S. Mirjalili, S. M. Mirjalili, and A. Lewis Grey Wolf Optimizer Advances in Engineering Software, vol. 69, pp. 46-61, 2014.

[19] B. J. Frey and D. Dueck Clustering by Passing Messages Between Data Points Science, 315:972-976, February 2007.

[20] A. Sallahi and M. St-Hilaire Optimal model for the controller placement problem in software defined networks IEEE Communication. Lett., vol. 19, no. 1, pp. 30-33, Jan. 2015.

[21] C. Wang, B. Hu, S. Chen, D. LI, AND B. LiU A switch migration-based decision-making scheme for balancing load in SDN IEEE Access, vol. 5, pp. 4537-4544, 2017.

[22] Liyanage, M.; Gurtov, A.;And Ylianttila M Software Defined Mobile Networks (SDMN): Beyond LTE Network Architecture JohnWiley \& Sons: Hoboken, NJ, USA, 2015.

[23] Lange, S.; Gebert, S.; Zinner, T.; Tran-Gia, P.; Hock, D.; Jarschel, M.; And Hoffmann, M.Heuristic approaches to the controller placement problem in large scale SDN networks IEEE Trans. Netw. Serv. Manag. 2015, 12,pp. 4-17.

[24] T. Koponen et AL. Onix: A distributed control platform for large-scale production networks in Proc. OSDI, vol. 10, 2010, pp. 351-364.

[25] A. TOOTOONChian ,AND Y. Ganjali Hyperflow: a distributed control plane for open- flow in: Internet Network Management Conference on Research on Enterprise NETWORKING, 2010 . pp. 3-3.

[26] A.R. Curtis, J.C. Mogul, J. Tourrilhes, P. Yalagandula, P. Sharma, and S. Banerjee Devoflow: scaling flow management for high-performance networks Acm Sig- comm Comput. Commun. Rev. 41 (4) (2011) pp. 254-265.

[27] R. Amin, M. Reisslein, and N. Shah Hybrid SDN networks: A survey of existing approaches IEEE Commun. Surveys Tuts., vol. 20, no. 4, pp. 3259-3306, 4th Quart., 2018.

[28] D. Hock ET AL. Pareto-optimal resilient controller placement in SDN based core networks in Proc. IEEE 25th Int. Teletraffic Congr. (ITC), 2013, pp. 1-9.

[29] S. Liu, H. Wang, S. Yi, and F. Zhu NCPSO: A solution of the controller placement problem in software defined networks in Proc. Int. Conf. Algorithms Archit. Parallel Process. Cham, Switzerland: Springer, 2015, pp. 213-225.

[30] N. Beheshti And Y. Zhang Fast fail over for control traffic in software defined networks in Proc. IEEE Global Commun. Conf., Dec. 2013, pp. 2665-2670.

[31] B. P. R. Killi, E. A. RedDy, AND S. V. Rao Cooperative game theory based network partitioning for controller placement in SDN in Proc. IEEE 10th Int. Conf. Commun. Syst. Netw. (COMSNETS), 2018, pp. 105-112.

[32] S.K. Keshari, V. Kansal, and S. Kumar, An Intelligent Way for Optimal Controller Placements in Software-Defined-IoT Networks for Smart Cities Journal of Computers \& Industrial Engineering, ISSN-0360-8352, pp. 107667, 2021.

[33] I. Benkacem, T. Taleb, M. BagaA, and H. Flinck Optimal VNFs placement in CDN slicing over multi-cloud environment IEEE J. Sel. Areas Commun., vol. 36, no. 3, pp. 616-627, Mar. 2018.

[34] T. TAleb, M. BagaA, AND A. Ksentini User mobility-aware virtual network function placement for virtual $5 G$ network infrastructure in Proc. IEEE Int. Conf. Commun. (ICC), 2015, pp. 3879-3884.

[35] M. BagaA, T. Taleb, And A. Ksentini Service-aware network function placement for efficient traffic handling in carrier cloud in Proc. IEEE Wireless Communication Network Conf. (WCNC), 2014, pp. 2402-2407.

[36] K. Atefi, S. Yahya, A. Rezaei, And A. Erfanian Traffic behavior of Local Area Network based on M/M/1 queuing model using poisson and exponential distribution in Proc. IEEE Region 10 Symp. (TENSYMP), May 2016, pp. 19-23.

[37] D. P. Bertsekas, R. G. Gallager, and P. Humblet Data Networks. Upper Saddle River, NJ, USA: Prentice-Hall, 1992.

Edited by: Pradeep Kumar Singh

Received: Jun 26, 2021

Accepted: Oct 8, 2021 
